\title{
Bacterial symbionts in agricultural systems provide a strategic source for antibiotic discovery
}

\author{
Timothy R Ramadhar ${ }^{1}$, Christine Beemelmanns ${ }^{1}$, Cameron R Currie ${ }^{2}$ and Jon Clardy ${ }^{1}$ \\ As increased antibiotic resistance erodes the efficacy of currently used drugs, the need for new candidates with therapeutic \\ potential grows. Although the majority of antibiotics in clinical use originated from natural products, mostly from environmental \\ actinomycetes, high rediscovery rates, among other factors, have diminished the enthusiasm for continued exploration of this \\ historically important source. Several well-studied insect agricultural systems have bacterial symbionts that have evolved to \\ produce small molecules that suppress environmental pathogens. These molecules represent an underexplored reservoir \\ of potentially useful antibiotics. This report describes the multilateral symbioses common to insect agricultural systems, the \\ general strategy used for antibiotic discovery and pertinent examples from three farming systems: fungus-farming ants, \\ southern pine beetles (SPBs) and fungus-growing termites.
}

The Journal of Antibiotics (2014) 67, 53-58; doi:10.1038/ja.2013.77; published online 7 August 2013

Keywords: agriculture; antibiotics; bacteria; fungus farming; insects; multilateral symbiosis; natural products

\section{INTRODUCTION}

For most people, agriculture conjures up an image of farmers tending their crops - the plants that provide food, fiber or biofuels for the rest of us. Humans started farming $\sim 10000$ years ago, but our efforts were preceded by other agriculture systems, as certain ants, beetles and termites have farmed their own food for millions of years. ${ }^{1}$ In these agricultural systems, the insect farmers cannot live without their crops, and the crops cannot live without the farmers-an arrangement referred to as an obligate mutualistic symbiosis. These arrangements are obligate because of the dependence, mutualistic because both partners benefit and symbiotic because the insects and crops colocalize. Maintaining an agricultural system requires protecting the crops from pathogens, and farmers use chemical defenses to safeguard their crops. Human farmers use small-molecule fungicides like azoxystrobilin, a synthetic molecule inspired by the natural product strobilurin, and bactericides like Agri-Mycin, whose active ingredient is streptomycin, to protect their crops. Insect farmers also use small-molecule control agents, along with other behavioral strategies, ${ }^{1-3}$ to ward off crop pathogens. The insects do not produce these compounds themselves; they enlist the help of bacterial symbionts to perform this crucial task. ${ }^{4-9}$ These bacterial symbionts, which expand the bilateral insect farmer-fungal crop symbioses to tripartite symbioses, produce antibiotic agents that suppress the growth of both specialist and generalist crop pathogens.

In modern human agriculture, crop loss results in reduced revenue for the farmers and food shortages for the consumers, but crop loss in an insect system has even more dire consequences. The bacteria providing the chemical defenses and the microbial pathogens selected to overcome them appear to have been coevolving for millions of years, and the molecules employed today are the products of multiple rounds of natural selection. These bacterially produced small molecules provide a remarkable pool of therapeutic candidates that we can exploit to control the microbial pathogens that threaten our well-being.

Insects have been using the same types of bacteria to produce useful antibiotics that humans have been using - they just appear to have been doing it much longer. A majority of the clinical antibacterial and antifungal agents come from natural products or their synthetic analogs, ${ }^{10-12}$ and historically soil actinomycetes have been the primary source. However, continued reliance on this source has led to unacceptably high rates of rediscovering previously identified compounds, and rediscovery, along with other factors, has led to greatly diminished interest in and resources for natural product discovery in pharmaceutical companies. ${ }^{13,14}$ The failure of replacement discovery strategies to significantly expand the antibiotic pipeline and the progressive expansion of resistance towards prescribed antibiotics have generated a sense of even greater urgency for the discovery of new antibiotic leads along with an increased willingness to explore other discovery strategies. ${ }^{12,15,16}$ Exploiting the bacterial symbionts in insect agricultural systems provides one approach. ${ }^{17,18}$ This approach has several attractive features, which are as follows: (1) it is a variation on historically

${ }^{1}$ Department of Biological Chemistry and Molecular Pharmacology, Harvard Medical School, Boston, MA, USA and ${ }^{2}$ Department of Bacteriology, University of WisconsinMadison, Madison, WI, USA

Correspondence: Professor J Clardy, Department of Biological Chemistry and Molecular Pharmacology, Harvard Medical School, 240 Longwood Avenue, Boston, MA 02115, USA. 
successful methods as only the source of the actinomycetes differs from earlier practices; (2) there are literally thousands of species that participate in agriculture, therefore, a highly efficient algorithmic approach can be employed and (3) the approach matches the evolutionary selection principle with the intended use: antibiotic activity. Below we describe early progress in developing a discovery pipeline, with illustrative examples from fungus-farming ants, southern pine beetles (SPBs) and fungus-growing termites.

\section{INSECT AGRICULTURAL MODEL AND STRATEGY FOR ALGORITHMIC ANTIBIOTIC DISCOVERY}

Although the origin of insect agricultural systems occurred at different times and in widely separated geographic locations, they have a common organizational structure (Figure 1). The insects use fungi to render hard-to-digest plant-based materials into an edible form. They provide the raw material, the fungi decompose it and the insects then consume the fungi. The insects benefit the fungus by providing it nutrients, and the fungus benefits the insects by returning the favor. Of course this fungal garden is attractive to consumers other than the insect farmer, and these exploiters threaten the fungal crop and indirectly the insect farmers. Frequently, the most troublesome predators are other fungi. The symbiotic bacteria, which are often carried by the insect farmers in specialized anatomical compartments, produce small molecules that suppress the growth of the predatory fungus while leaving the growth of the crop fungus largely untouched. The insect confers a benefit on the bacteria by moving it from place to place, and the bacteria confer a benefit on the farmer and the crop through the well-known ecological relationship: 'the enemy of my enemy is my friend'. This overall strategy has clearly succeeded. The ant system originated with a single species some 50 million years ago somewhere in the Amazon basin, and today fungus-farming ants total over 200 species and are the major herbivores in the New World Tropics. ${ }^{19}$ Bark beetles, which originated a minimum of 100 million years ago, ${ }^{20}$ have expanded to 220 genera and over 6000 species, ${ }^{21}$ whereas fungus-growing termites, which occupy the same ecological niche in the Old World Tropics that the fungus-farming ants occupy in the New World Tropics, originated around 30 million years ago and have evolved into more than 300 species. $^{22,23}$ These insect farmers follow the general strategy outlined in Figure 1, but they do so with different tactics as their crops and crop predators differ. Successfully addressing these differences generated the multiple bacterial strains that afford the evolutionarily selected small molecules available today.

Identifying the biologically active small molecules typically begins with the techniques of bioassay-guided fractionation and dereplication. ${ }^{24}$ The bacteria are isolated from either specialized anatomical

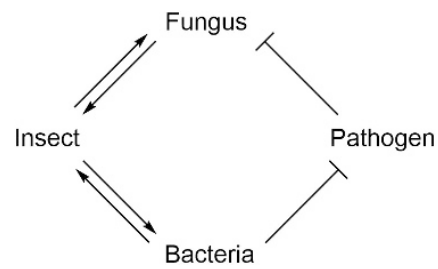

Figure 1 Symbiotic interactions within a generalized insect agricultural system. Arrows indicate a benefit and T-bars indicate suppression. A mutualistic symbiosis exists between the fungus and the insect. The fungus is food for the insect, and the insect provides raw materials for the fungus. The pathogen threatens the fungus, which in turn threatens the insect. The mutualistic bacteria produce antibiotics to suppress the pathogen. The insect provides nutrients and transports the bacteria. compartments, crypts in the case of ants and mycangia in the case of SPBs, or the termite nests. They are cultured under a variety of conditions, including co-culturing with microbial co-habitants and pathogens, ${ }^{25}$ and culture extracts are tested against either the specialist predator or a suitable surrogate. Secondary assays against the crop fungus are employed to ensure that the assay is ecologically relevant, and additional secondary assays against human pathogens or cell lines derived from human cancers can indicate therapeutically relevant activities. The large number of bacterial producers available from these insect agriculture systems provides the impetus to set up streamlined moderate-throughput discovery processes and also empowers differential metabolomic approaches to discovery (for example, dereplication), which will be illustrated by a few examples. In addition to the traditional bioassay-guided fractionation approach, the low cost of genome sequencing and the growing power of genome mining have led to the discovery of additional biologically active small molecules.

\section{FUNGUS-GROWING ANTS}

Attine ants have participated in agricultural practices for over 45 million years starting with a single origin in the Amazon rainforest. ${ }^{1,3,19}$ They are capable of cultivating various fungi (for example, Basidiomycota such as Agaricales: Lepiotaceae and Pterulaceae) as the main food source of the larvae and adults. Individual colonies cultivate a single crop fungus that is transmitted vertically by the daughter queens as they carry small pellets of nest mycelium in their infrabuccal pockets, which are pouches in the ant's oral cavity, when they leave their parent colony to establish new nests. The balance between the ants and crop fungi can be threatened, however, when invasive fungi attempt to outcompete the crop fungi. In particular, Escovopsis spp., a pathogenic microfungus specialized on this ant-fungus mutualism, can infiltrate and consume the ants' fungal garden. ${ }^{4,26-28}$ However, the long-term success of the obligate relationship between the Attine ant and its crop fungus indicates that the ants have devised a strategy that is able to counterbalance infections of Escovopsis over evolutionary time.

Scanning electron microscopic studies by Currie et al. ${ }^{4,26,29}$ demonstrated that Attine ants are coated with filamentous actinomycete bacteria, belonging to the genus Pseudonocardia, ${ }^{30,31}$ and that these bacteria produce diffusible small molecules that are capable of inhibiting the growth of Escovopsis spp. These findings indicate a tripartite mutualistic relationship between the ants, the food fungus and Pseudonocardia spp. (Figure 2). The relationship between the ants and Pseudonocardia spp. is so important to the

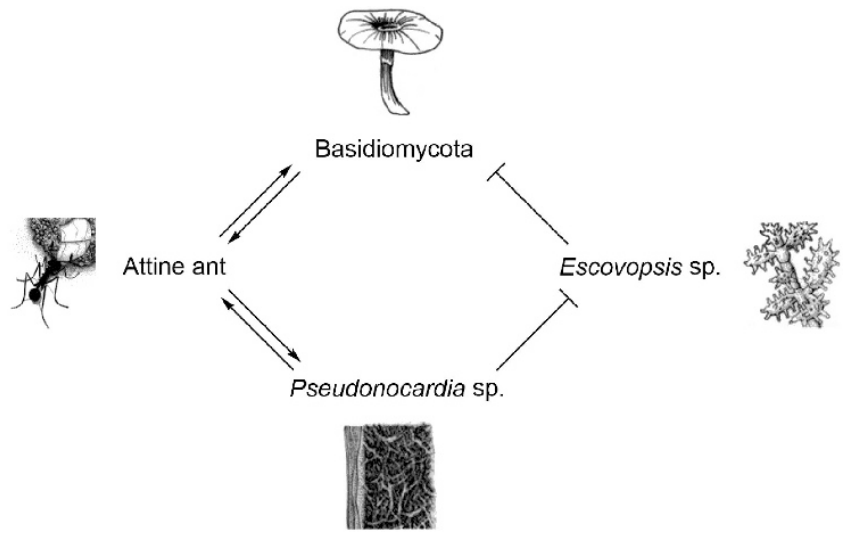

Figure 2 Symbiotic interactions in the Attine ant agricultural system. 
success of the agricultural system that the ants have evolved specialized cuticular crypts and exocrine glands to house and nourish the bacteria. ${ }^{32}$

A collaborative study by the Clardy and Currie laboratories was initiated to analyze the molecular basis of this highly successful tripartite symbiosis. ${ }^{6}$ The study first involved an Escovopsis spp. and a Pseudonocardia spp. from the nest of an ant (Apterostigma dentigerum) collected in Panama. A pairwise challenge assay was performed to verify the ability of the Pseudonocardia strain to inhibit the Escovopsis isolate. Next, the Pseudonocardia strain was cultured on an $8 \mathrm{~L}$ scale in YMEA (yeast extract, malt extract and glucose) liquid medium and was extracted with EtOAc. Bioassay-guided fractionation of the crude material was employed using $\mathrm{C} 18$ resin and an assay against Escovopsis sp. to identify the relevant fractions, which were further purified using reversed-phase HPLC with a C18 semipreparative column. Ultimately, a selective antifungal agent against the Escovopsis isolate was identified and assigned the name dentigerumycin $\left(\mathrm{C}_{40} \mathrm{H}_{67} \mathrm{~N}_{9} \mathrm{O}_{13}\right)$ (Figure 3), which was derived from the name of the ant (A. dentigerum) from which the Pseudonocardia bacterial strain was isolated.

Dentigerumycin is a cyclic depsipeptide that contains unusual amino acids ( $\beta$-hydroxyleucine, $N$-hydroxyalanine, piperazic acid and $\gamma$-hydroxypiperazic acid) and a pyran side chain. ${ }^{6}$ The biosynthesis of dentigerumycin likely involves a hybrid polyketide synthase (PKS)/ non-ribosomal peptide synthase (NRPS) gene cluster. The relative stereochemistry for the pyran ring was determined through ROESY NMR experiments, and the absolute stereochemistry of dentigerumycin was determined through a combination of advanced Marfey analysis, ${ }^{33}$ modified Mosher analysis ${ }^{34}$ and CD spectroscopy. Dentigerumycin has some similarity with polyoxypeptins ${ }^{35}$ and aurantimycins, ${ }^{36}$ as they share some common amino acids; however, the core structures are different. Bioassays with purified dentigerumycin demonstrated that it was able to inhibit the Escovopsis isolate (MIC: $2.8 \mu \mathrm{m}$ ) without significantly affecting the Basidiomycota cultivar. Dentigerumycin also exhibited antifungal activity against various strains of Candida albicans (MIC: $1.1 \mu \mathrm{M}$ ). Additionally, it was selectively cytotoxic against the National Institutes of Health (NIH) National Cancer Institute's (NCI) panel of human cancer cell lines (unpublished data). This last result indicates that the selectivity imposed by the system - the ability to suppress growth in a fast-growing eukaryote (Escovopsis) is much more than the growth of a slow-growing eukaryote (crop fungus) — could also afford molecules with therapeutic potential against cancer.

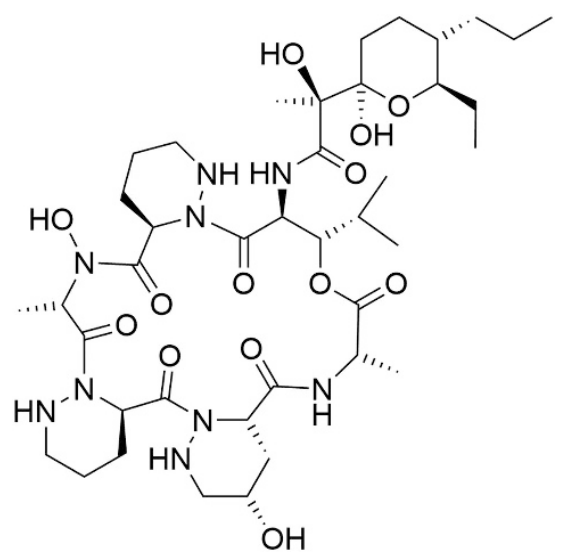

Figure 3 Structure of dentigerumycin.
Dentigerumycin is not the only antifungal agent demonstrated to have activity against Escovopsis sp. A similar discovery approach was undertaken by Spiteller and co-workers ${ }^{37}$ to identify the Escovopsis sp. antifungal agent produced by various Streptomyces sp. that they isolated from leaf-cutter ants in the genus Acromyrmex. They were able to determine through bioassay-guided fractionation and dereplication that known candicidin macrolides have antifungal activity against Escovopsis sp. without having significant effect on the Leucoagaricus gongylophorus food fungus. Further studies by Spiteller and co-workers ${ }^{38}$ led to the isolation of known actinomycins, antimycins and valinomycins from Streptomyces associated with leaf-cutting ants. Other recent studies on fungusgrowing ant systems have resulted in the identification of nystatin P1 from a Pseudonocardia sp. isolated from Acromyrmex octospinosus, ${ }^{39}$ novel pseudonocardones A-C and known antibiotics 6-deoxy-8-Omethylrabelomycin and X-14881 E from Pseudonocardia sp. EC080529-01, ${ }^{40}$ and there is potential for isolating additional smallmolecule antibiotics from Streptomyces S4. ${ }^{41}$ It is clear that further research will be needed in order to fully elucidate the complex interactions within these ant systems and the roles of small molecules in maintaining them, with concomitant determination of their therapeutic potential.

\section{SOUTHERN PINE BEETLES}

The SPB, Dendroctonus frontalis, which is currently responsible for the massive destruction of pine trees that created monetary losses and changes in the local environment, participates in a mutualism with the fungus Entomocorticium sp. A, which serves as a larval food source. ${ }^{42,43}$ An adult SPB excavates the inner bark and phloem of pine trees to form ovipositional galleries. The SPB inoculates the gallery walls during the excavation process with Entomocorticium sp. A, which is transmitted in storage compartments on the beetle known as mycangia. The female beetles then breed with the males inside the galleries and lay their fertilized eggs. Once the larvae have developed into adults, they emerge from the galleries and spread to other pine trees to repeat the cycle. However, the D. frontalis-Entomocorticium sp. A symbiosis is exploited by the Ophiostoma minus fungus. This pathogen is a food source for Tarsonemus sp., which is a phoretic mite that hitchhikes on the exoskeleton of $D$. frontalis. When $D$. frontalis forms its ovipositional galleries, Tarsonemus sp. inoculates the galleries with the O. minus that covers its exoskeleton. O. minus can normally outgrow Entomocorticium sp. A, which leads to a devastating outcome for the SPB larvae. Like the ant-fungal systems, the D. frontalisEntomocorticium sp. A relationship is expected to have evolved defensive strategies to counteract the threat from $O$. minus.

A concerted effort between the Yuceer, Klepzig, Clardy and Currie laboratories was undertaken to probe the nature of selective O. minus inhibition (Figure 4). ${ }^{5,44}$ Scanning electron microscopy studies of the ovipositional galleries and inside the SPB mycangium resulted in the unexpected observation of rampant actinomycete growth. Subsequent

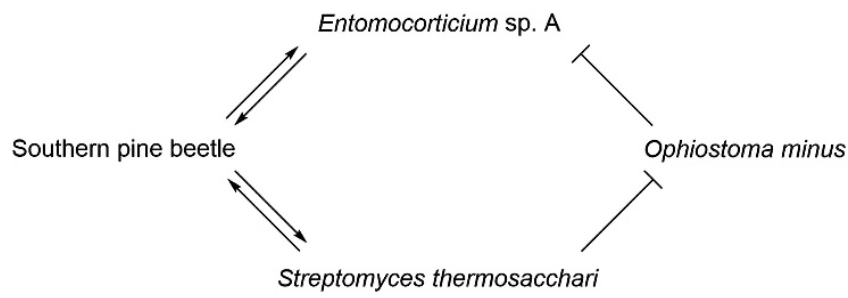

Figure 4 Symbiotic interactions in the southern pine beetle agricultural system. 
isolation experiments led to the identification of two actinomycete morphotypes, one red and one white. Both were determined to be part of the monophyletic clade related to Streptomyces thermosacchari through DNA sequencing and 16S rDNA analysis. The red and white Streptomyces morphotypes were referred to as SPB74 and SPB78, respectively.

Pairwise challenge bioassays on yeast malt extract agar demonstrated that the red morphotype SPB74 bacterium inhibited the growth of $O$. minus, whereas having only a slight effect on Entomocorticium sp. A growth. A suitable set of growth conditions for scale-up were determined using YMEA and YPM (yeast extract, peptone and mannitol) liquid media. EtOAc extraction of the liquid medium followed by fractionation using C18 stationary phase and hexane partitioning afforded a polyene peroxide, which was given the name mycangimycin $\left(\mathrm{C}_{20} \mathrm{H}_{24} \mathrm{O}_{4}\right)$ (Figure 5) based on the mycangium where SPB74 was isolated. Mycangimycin is light sensitive, decomposes at high concentrations and behaves poorly on silica or C18 resins. ${ }^{44}$ It contains a conjugated heptaene, carboxylic acid and a 1,2dioxolane moiety. The syn substitution pattern of the dioxolane was determined through a ROESY NMR experiment, verification of the peroxide functionality was performed by scission of the peroxyl O-O bond through $\mathrm{Zn} / \mathrm{AcOH}$ reduction of mycangimycin methyl ester (formed through reaction with TMS-diazomethane) and absolute stereoconfiguration was established on the resultant syn 1,3-diol through a modified Mosher procedure. ${ }^{45}$

Mycangimycin was found to have an antifungal activity against Saccharomyces cerevisiae and various $C$. albicans strains with a similar level of potency observed against O. minus (MIC ranges: $\left.0.4-2.5 \mu \mathrm{g} \mathrm{ml}^{-1}\right){ }^{44}$ Additionally, as mycangimycin and some antimalarial drugs (for example, artemisinin ${ }^{46}$ and synthetic 1,3,5trioxolanes ${ }^{47}$ ) share peroxyl moieties, ${ }^{48}$ mycangimycin was tested in an antimalarial assay against Plasmodium falciparum. ${ }^{49}$ An $\mathrm{EC}_{50}$ of $17 \mathrm{ng} \mathrm{ml}^{-1}$ was observed for mycangimycin, which is very similar to the $\mathrm{EC}_{50}$ values of other clinical antimalarial drugs such as artemisinin, chloroquine, mefloquine and pyrimethamine, which have $\mathrm{EC}_{50}$ values in the range of $10 \mathrm{ng} \mathrm{ml}^{-1}$.

Whereas SPB74 had inhibitory activity against O. minus in the pairwise challenge bioassay, no activity was observed with SPB78 (white Streptomyces morphotype). However, upon further studies through a differential metabolomics strategy, SPB78 was found to produce novel antifungal compounds. ${ }^{50}$ SPB78 was subjected to various growth conditions, and cultivation on YMS (yeast extract, malt extract and soluble starch) agar plates led to the production of new metabolites as observed through LC-MS. Subsequent scale-up, EtOAc extraction, fractionation through $\mathrm{C} 18$ resin and semipreparative HPLC on a C18 column led to the isolation of two new polycyclic tetramate macrolactams: frontalamides $A\left(\mathrm{C}_{29} \mathrm{H}_{36} \mathrm{~N}_{2} \mathrm{O}_{7}\right)$ and $\mathrm{B}\left(\mathrm{C}_{29} \mathrm{H}_{36} \mathrm{~N}_{2} \mathrm{O}_{6}\right)$ (Figure 6). They were named after the SPB (D. frontalis) from which SPB78 was isolated. Both frontalamides $A$ and $B$ exhibit structural similarity with alteramide $A,{ }^{51}$ cylindramide, ${ }^{52}$ discodermide, ${ }^{53}$ ikarugamycin, ${ }^{54}$ maltophilin ${ }^{55}$ and

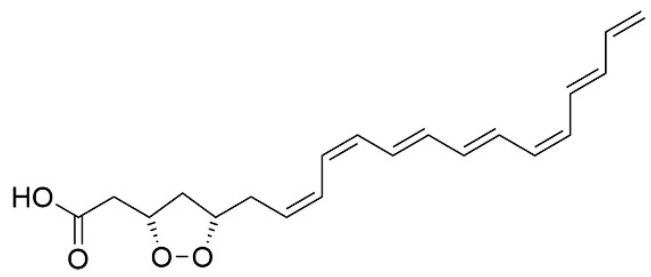

Figure 5 Structure of mycangimycin. dihydromaltophilin. ${ }^{56}$ The biosynthetic cluster for the frontalamides was identified and a mutant in which one of the genes was deleted led to the isolation of structurally related polycyclic tetramate macrolactam compounds. A pairwise challenge bioassay between O. minus and SPB78 performed on a YMS agar plate identified that the frontalamides have antifungal activity against $O$. minus. This case emphasizes the importance of employing both a variety of growth conditions and bioinformatic analysis in order to fully explore the bacterial metabolome.

Subsequent genomic mining studies with the frontalamide biosynthetic gene cluster in the same report revealed that other actinobacteria and proteobacteria carry similar clusters. A screen of environmental actinomycete isolates using a degenerate primer-based PCR method was employed in order to detect a conserved sequence of the frontalamide gene cluster. This experiment successfully identified isolates that carry frontalamide-like clusters. Expression studies indicated that these isolates produced polycyclic tetramate macrolactam metabolites. A subsequent study in the Clardy laboratory reported the isolation of new polycyclic tetramate macrolactams, named clifednamides $\mathrm{A}\left(\mathrm{C}_{29} \mathrm{H}_{36} \mathrm{~N}_{2} \mathrm{O}_{5}\right)$ and $\mathrm{B}\left(\mathrm{C}_{29} \mathrm{H}_{36} \mathrm{~N}_{2} \mathrm{O}_{6}\right)$, from Streptomyces sp. JV178 (Figure 6). ${ }^{57}$ Therefore, the potential to isolate more frontalamide analogs clearly exists, which can allow for structure activity relationship insights into this class of antifungal agents.

\section{FUNGUS-GROWING TERMITES}

Fungus-growing termites (Isoptera) participate in agricultural practices by cultivating Termitomyces. The termites gather a pile of fecal deposits that contain plant material to form a comb that Termitomyces is able to degrade. ${ }^{58,59}$ The fungi then produce nodules (primordial fruiting bodies) that are consumed by the termites, in addition to the digesting parts of the fungal comb, for nourishment. The fungal cells from the nodules survive passage through the termite's digestive system and inoculate other fecal combs crafted

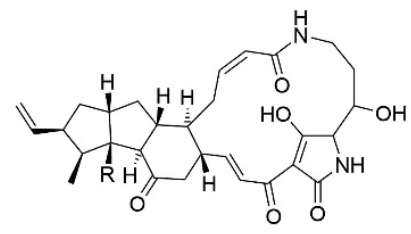

frontalamide $A: R=O H$ frontalamide $B: R=H$
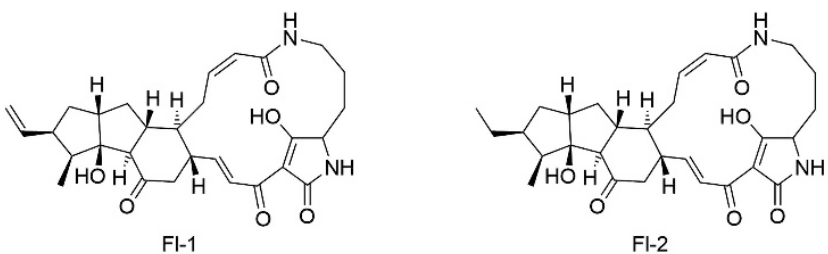

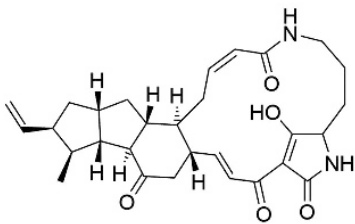

$\mathrm{Fl}-3$

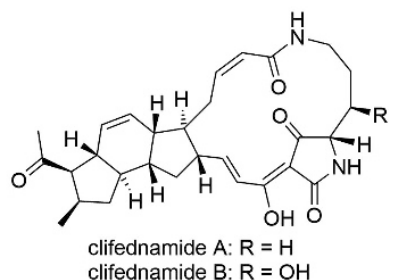

Figure 6 Structures of frontalamides $A$ and $B$ and their biosynthetic analogs, and clifednamides $A$ and $B$. 
by the termites. Nodules that are not consumed by the termite develop into sexual basidiospores, which are wind-distributed to other locales and serve as the inoculum for new fungal gardens in other termite colonies. ${ }^{60}$ This mutualistic relationship has an important role in the degradation of plant matter in Southeast Asia and Sub-Saharan Africa. ${ }^{61-64}$ However, the growth of Termitomyces can be negatively perturbed by a competitor fungus (Pseudoxylaria), ${ }^{65-67}$ whose fruiting bodies frequently occur in abandoned termite nests ${ }^{68,69}$ and is known to outcompete Termitomyces fungal gardens when no termites are present. $66,70,71$

In an analogous manner to fungus-growing ants and SPBs, fungusgrowing termites may have evolved symbiotic strategies to suppress the growth of pathogenic fungi, such as Pseudoxylaria, within their nests. A recent report from Visser et al. ${ }^{72}$ described efforts to search for symbiotic bacteria that exert selective antifungal activity against Pseudoxylaria. Actinobacteria were found throughout all 30 sampled fungus-growing nests and colonies from isolation studies, and phylogenetic analysis revealed that they are interspersed with strains that originate from species other than fungus-growing termites. Pairwise challenge bioassays indicated that many of the actinobacteria were able to produce antifungal agents that inhibit Pseudoxylaria; however, they also inhibit Termitomyces. These observations are compatible with the following possibilities: (1) no defensive symbionts are present in the system, (2) defensive symbionts represent a subset of the isolated community that have not been identified or successfully cultivated ex situ, (3) the growth conditions in the pairwise challenge assay did not elicit expression of selective antifungal agents or (4) the non-selective antifungal compounds are directly applied to infected areas within the fungal garden by the termites. Further research by the Clardy and Currie laboratories is currently exploring the nature of the termite agricultural relationship and the discovery of selective antifungal agents from these systems.

A preliminary study in the Clardy, Currie and Bugni laboratories described a metabolomics strategy based on dereplication to find new secondary metabolites from a fungus-growing termite-associated Streptomyces. ${ }^{73}$ A total of 30 Streptomyces spp. were initially analyzed and one Streptomyces sp. (MspM5) was determined to produce novel compounds upon performing principal component analysis of LC-MS traces and querying the high-resolution mass in<smiles></smiles>

microtermolide A

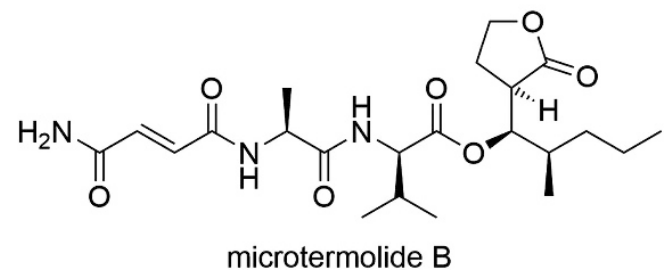

Figure 7 Structures of microtermolides $\mathrm{A}$ and $\mathrm{B}$.
Antibase. Scale-up culturing of MspM5 was performed on solid ISP-2 medium. The solid medium was extracted with EtOAc, and purified using standard and HPLC chromatography using $\mathrm{C} 18$ resin to afford microtermolides $\mathrm{A}\left(\mathrm{C}_{23} \mathrm{H}_{37} \mathrm{~N}_{3} \mathrm{O}_{6}\right)$ and $\mathrm{B}\left(\mathrm{C}_{22} \mathrm{H}_{35} \mathrm{~N}_{3} \mathrm{O}_{7}\right)$ (Figure 7). Both secondary metabolites are depsipeptides that are proposed to be biosynthesized through hybrid PKS-NRPS pathways, and microtermolide B is a member of the rare class of linear depsipeptides, which includes several cyanobacteria-produced compounds: dolastatin $15,{ }^{74}$ gallinamide $\mathrm{A},{ }^{75}$ symplocin $\mathrm{A}^{76}$ and symplostatin $3 .{ }^{77}$ Microtermolide $\mathrm{B}$ appears to be the first example of a linear depsipeptide produced by a Streptomyces sp. It is unclear whether microtermolide B is a precursor to microtermolide A, and if so, whether this transformation requires enzyme catalysis. Neither microtermolides had activity against Bacillus subtilis 3610 or S. cerevisiae; however, studies are underway in the Clardy laboratory to determine activity of these compounds towards Pseudoxylaria and other termite crop pathogens.

\section{CONCLUDING REMARKS}

Insect agricultural systems provide a very promising source for discovering novel antibiotics produced by the associated bacterial symbionts as illustrated by the aforementioned examples. It is clear that continued study of the symbiotic bacteria that maintain robust insect-farming systems can afford undiscovered molecules with therapeutic potential. These studies will also address basic scientific questions because they place naturally occurring small molecules in their ecologically and evolutionarily relevant contexts. The only reason that the minuscule fraction of small molecules that occur naturally have any special significance is their evolutionary history, the numerous rounds of mutation, selection and expansion that have shaped the molecules that can be found today. Ironically, we know little about how biosynthetic pathways evolved or how molecules were selected. The molecules produced by the bacterial symbionts of insect agricultural systems can address these questions, as both the phylogeny of the bacteria and their intended purpose are reasonably clear.

\section{ACKNOWLEDGEMENTS}

We thank the members of the Clardy and Currie laboratories who are engaged in this project. We are also grateful for financial support through the National Institutes of Health (NIH) (R01 GM086258 to J.C. and GM096347 to C.R.C.), through the National Science Foundation (NSF) (MCB-0702025) to C.R.C., and through the German National Academy of Sciences Leopoldina via a postdoctoral fellowship to C.B.

1 Mueller, U. G., Gerardo, N. M., Aanen, D. K., Six, D. L. \& Schultz, T. R. The evolution of agriculture in insects. Annu. Rev. Ecol. Evol. Syst. 36, 563-595 (2005).

2 Currie, C. R. \& Stuart, A. E. Weeding and grooming of pathogens in agriculture by ants. Proc. R. Soc. Lond. B 268, 1033-1039 (2001).

3 Currie, C. R. A community of ants, fungi, and bacteria: a multilateral approach to studying symbiosis. Annu. Rev. Microbiol. 55, 357-380 (2001).

4 Currie, C. R., Scott, J. A., Summerbell, R. C. \& Malloch, D. Fungus-growing ants use antibiotic-producing bacteria to control garden parasites. Nature 398, 701-704 (1999).

5 Scott, J. J. et al. Bacterial protection of beetle-fungus mutualism. Science 322, 63-63 (2008).

6 Oh, D.-C., Poulsen, M., Currie, C. R. \& Clardy, J. Dentigerumycin: a bacterial mediator of an ant-fungus symbiosis. Nat. Chem. Biol. 5, 391-393 (2009).

7 Kaltenpoth, M. Actinobacteria as mutualists: general healthcare for insects? Trends Microbiol. 17, 529-535 (2009).

8 Brownlie, J. C. \& Johnson, K. N. Symbiont-mediated protection in insect hosts. Trends Microbiol. 17, 348-354 (2009).

9 Seipke, R. F., Kaltenpoth, M. \& Hutchings, M. I. Streptomyces as symbionts: an emerging and widespread theme? FEMS Microbiol. Rev. 36, 862-876 (2012).

10 Clardy, J. \& Walsh, C. Lessons from natural molecules. Nature 432, 829-837 (2004).

11 Clardy, J., Fischbach, M. A. \& Walsh, C. T. New antibiotics from bacterial natural products. Nat. Biotech. 24, 1541-1550 (2006). 
12 Clardy, J., Fischbach, M. A. \& Currie, C. R. The natural history of antibiotics. Curr. Biol. 19, R437-R441 (2009).

13 Baltz, R. H. Marcel Faber Roundtable: is our antibiotic pipeline unproductive because of starvation, constipation or lack of inspiration? J. Ind. Microbiol. Biotech. 33 507-513 (2006).

$14 \mathrm{Li}$, J. W.-H. \& Vederas, J. C. Drug discovery and natural products: end of an era or an endless frontier? Science 325, 161-165 (2009).

15 Fischbach, M. A. \& Walsh, C. T. Antibiotics for emerging pathogens. Science 325, 1089-1093 (2009).

16 Walsh, C. \& Fischbach, M. New ways to squash superbugs. Sci. Am. 301, 44-51 (2009).

17 Bode, H. B. Insects: true pioneers in anti-infective therapy and what we can learn from them. Angew. Chem. Int. Ed. 48, 6394-6396 (2009).

18 Crawford, J. M. \& Clardy, J. Bacterial symbionts and natural products. Chem. Commun. 47, 7559-7566 (2011).

19 Schultz, T. R. \& Brady, S. G. Major evolutionary transitions in ant agriculture. Proc. Natl. Acad. Sci. USA 105, 5435-5440 (2008).

20 Cognato, A. I. \& Grimaldi, D. 100 million years of morphological conservation in bark beetles (Coleoptera: Curculionidae: Scolytinae). Sys. Entomol. 34, 93-100 (2009).

21 Keeling, C. I. et al. Draft genome of the mountain pine beetle, Dendroctonus ponderosae Hopkins, a major forest pest. Genome. Biol. 14, R27 (2013).

22 Aanen, D. K. et al. The evolution of fungus-growing termites and their mutualistic fungal symbionts. Proc. Natl Acad. Sci. USA 99, 14887-14892 (2002).

23 Nobre, T., Koné, N. A., Konaté, S., Linsenmair, K. E. \& Aanen, D. K. Dating the fungus-growing termites' mutualism shows a mixture between ancient codiversification and recent symbiont dispersal across divergent hosts. Mol. Ecol. 20, 2619-2627 (2011).

24 Bugni, T. S., Harper, M. K., McCulloch, M. W. \& Whitson, E. L. in Natural Product Chemistry for Drug Discovery (eds Neidle, S., Buss, A. D. \& Butler, M. S.) 272-298 (Royal Society of Chemistry, Cambridge, 2009).

25 Seyedsayamdost, M. R., Traxler, M. F., Clardy, J. \& Kolter, R. Old meets new: using interspecies interactions to detect secondary metabolite production in actinomycetes. Methods Enzymol. 517, 89-109 (2012).

26 Currie, C. R., Mueller, U. G. \& Malloch, D. The agricultural pathology of ant fungus gardens. Proc. Natl Acad. Sci. USA 96, 7998-8002 (1999).

27 Currie, C. R. et al. Ancient tripartite coevolution in the attine ant-microbe symbiosis. Science 299, 386-388 (2003).

28 Reynolds, H. T. \& Currie, C. R. Pathogenicity of Escovopsis weberi: the parasite of the attine ant-microbe symbiosis directly consumes the ant-cultivated fungus. Mycologia 96, 955-959 (2004).

29 Currie, C. R., Bot, A. N. M. \& Boomsma, J. J. Experimental evidence of a tripartite mutualism: bacteria protect ant fungus gardens from specialized parasites. Oikos 101, 91-102 (2003)

30 Cafaro, M. J. \& Currie, C. R. Phylogenetic analysis of mutualistic filamentous bacteria associated with fungus-growing ants. Can. J. Microbiol. 51, 441-446 (2005).

31 Cafaro, M. J. et al. Specificity in the symbiotic association between fungus-growing ants and protective Pseudonocardia bacteria. Proc. R. Soc. B 278, 1814-1822 (2011).

32 Currie, C. R., Poulsen, M., Mendenhall, J., Boomsma, J. J. \& Billen, J. Coevolved crypts and exocrine glands support mutualistic bacteria in fungus-growing ants. Science 311, 81-83 (2006)

33 Fujii, K., Ikai, Y., Oka, H., Suzuki, M. \& Harada, K. A nonempirical method using LC/MS for Determination of the absolute configuration of constituent amino acids in a peptide: combination of Marfey's method with mass spectrometry and its practical application. Anal. Chem. 69, 5146-5151 (1997).

34 Seco, J. M., Quiñoá, E. \& Riguera, R. A practical guide for the assignment of the absolute configuration of alcohols, amines and carboxylic acids by NMR. Tetrahedron: Asymmetry 12, 2915-2925 (2001).

35 Umezawa, K., Nakazawa, K., Ikeda, Y., Naganawa, H. \& Kondo, S. Polyoxypeptins A and B produced by Streptomyces: apoptosis-inducing cyclic depsipeptides containing the novel amino acid (2S,3R)-3-Hydroxy-3-methylproline. J. Org. Chem 64, 3034-3038 (1999)

36 Gräfe, U. et al. Aurantimycins, new depsipeptide antibiotics from Streptomyces aurantiacus IMET 43917. Production, isolation, structure elucidation, and biological activity. J. Antibiot. 48, 119-125 (1995).

37 Haeder, S., Wirth, R., Herz, H. \& Spiteller, D. Candicidin-producing Streptomyces support leaf-cutting ants to protect their fungus garden against the pathogenic fungus Escovopsis. Proc. Natl Acad. Sci. USA 106, 4742-4746 (2009).

38 Schoenian, I. et al. Chemical basis of the synergism and antagonism in microbial communities in the nests of leaf-cutting ants. Proc. Natl Acad. Sci. USA 108 1955-1960 (2011)

39 Barke, J. et al. A mixed community of actinomycetes produce multiple antibiotics for the fungus farming ant Acromyrmex octospinosus. BMC Biol. 8, 109 (2010).

40 Carr, G., Derbyshire, E. R., Caldera, E., Currie, C. R. \& Clardy, J. Antibiotic and antimalarial quinones from fungus-growing ant-associated Pseudonocardia sp. J. Nat Prod. 75, 1806-1809 (2012).

41 Seipke, R. F. et al. A single Streptomyces symbiont makes multiple antifungals to support the fungus farming ant Acromyrmex octospinosus. PLoS One 6, e22028 (2011).

42 Klepzig, K. D. \& Wilkens, R. T. Competitive interactions among symbiotic fungi of the southern pine beetle. Appl. Env. Microbiol. 63, 621-627 (1997).

43 Hofstetter, R. W., Cronin, J. T., Klepzig, K. D., Moser, J. C. \& Ayres, M. P. Antagonisms mutualisms and commensalisms affect outbreak dynamics of the southern pine beetle. Oecologia 147, 679-691 (2006).
44 Oh, D.-C., Scott, J. J., Currie, C. R. \& Clardy, J. Mycangimycin, A polyene peroxide from a mutualist Streptomyces sp. Org. Lett. 11, 633-636 (2009).

45 Freire, F., Seco, J. M., Quiñoá, E. \& Riguera, R. Determining the absolute stereochemistry of secondary/secondary diols by $1 \mathrm{H}$ NMR: basis and applications. J. Org. Chem. 70, 3778-3790 (2005).

46 Eckstein-Ludwig, U. et al. Artemisinins target the SERCA of Plasmodium falciparum. Nature 424, 957-961 (2003).

47 Vennerstrom, J. L. et al. Identification of an antimalarial synthetic trioxolane drug development candidate. Nature 430, 900-904 (2004)

48 Tang, Y., Dong, Y. \& Vennerstrom, J. L. Synthetic peroxides as antimalarials. Med. Res. Rev. 24, 425-448 (2004).

49 Baniecki, M. L., Wirth, D. F. \& Clardy, J. High-throughput Plasmodium falciparum growth assay for malaria drug discovery. Antimicrob. Agents Chemother. 51, 716-723 (2007).

50 Blodgett, J. A. V. et al. Common biosynthetic origins for polycyclic tetramate macrolactams from phylogenetically diverse bacteria. Proc. Natl Acad. Sci. USA 107, 11692-11697 (2010).

51 Shigemori, H., Bae, M.-A., Yazawa, K., Sasaki, T. \& Kobayashi, J. Alteramide A, a new tetracyclic alkaloid from a bacterium Alteromonas sp. associated with the marine sponge Halichondria okadai. J. Org. Chem. 57, 4317-4320 (1992).

52 Kanazawa, S., Fusetani, N. \& Matsunaga, S. Cylindramide: Cytotoxic tetramic acid lactam from the marine sponge Halichondria cylindrata Tanita \& Hoshino. Tetrahedron Lett. 34, 1065-1068 (1993).

53 Gunasekera, S., Gunasekera, M. \& McCarthy, P. Discodermide: a new bioactive macrocyclic lactam from the marine sponge Discodermia dissoluta. J. Org. Chem. 56, 4830-4833 (1991).

54 Jomon, K., Kuroda, Y., Ajisaka, M. \& Sakai, H. A new antibiotic, ikarugamycin. J. Antibiot. 25, 271-280 (1972).

55 Jakobi, M. et al. Maltophilin: a new antifungal compound produced by Stenotrophomonas maltophilia R3089. J. Antibiot. 49, 1101-1104 (1996).

56 Graupner, P. R. et al. Dihydromaltophilin; a novel fungicidal tetramic acid containing metabolite from Streptomyces sp. J. Antibiot. 50, 1014-1019 (1997).

57 Cao, S., Blodgett, J. A. V. \& Clardy, J. Targeted discovery of polycyclic tetramate macrolactams from an environmental Streptomyces strain. Org. Lett. 12, 4652-4654 (2010).

58 Leuthold, R. H., Badertscher, S. \& Imboden, H. The inoculation of newly formed fungus comb with Termitomyces in Macrotermes colonies (Isoptera, Macrotermitinae). Insectes Soc. 36, 328-338 (1989).

59 Wood, T. G. \& Thomas, R. J. in Insect-fungus Interactions (eds Wilding, N., Collins, N. M., Hammond, P. M. \& Webber, J. F.) 69-92 (Academic Press, London, 1989).

60 Aanen, D. K. As you reap, so shall you sow: coupling of harvesting and inoculating stabilizes the mutualism between termites and fungi. Biol. Lett. 2, 209-212 (2006).

61 Lepage, M. Distribution, density and evolution of Macrotermes bellicosus nests (Isoptera: Macrotermitinae) in the north-east of ivory coast. J. Anim. Ecol. 53, 107-117 (1984)

62 Jones, J. A. Termites, soil fertility and carbon cycling in dry tropical Africa: a hypothesis. J. Trop. Ecol. 6, 291-305 (1990).

63 Mando, A. \& Brussaard, L. Contribution of termites to the breakdown of straw under Sahelian conditions. Biol. Fert. Soils 29, 332-334 (1999).

64 Konaté, S., Le Roux, X., Verdier, B. \& Lepage, M. Effect of underground fungus-growing termites on carbon dioxide emission at the point-and landscape-scales in an African savanna. Funct. Ecol. 17, 305-314 (2003).

65 Guedegbe, H. J., Miambi, E., Pando, A., Houngnandan, P. \& Rouland-Lefevre, C. Molecular diversity and host specificity of termite-associated. Xylaria. Mycologia 101, 686-691 (2009).

66 Visser, A. A. et al. Levels of specificity of Xylaria species associated with fungusgrowing termites: a phylogenetic approach. Mol. Ecol. 18, 553-567 (2009).

67 Hsieh, H.-M. et al. Phylogenetic status of Xylaria subgenus Pseudoxylaria among taxa of the subfamily Xylarioideae (Xylariaceae) and phylogeny of the taxa involved in the subfamily. Mol. Phylogenet. Evol. 54, 957-969 (2010).

68 Rogers, J. D. Thoughts and musings on tropical Xylariaceae. Mycol. Res. 104, 1412-1420 (2000)

69 Rogers, J. D., Ju, Y.-M. \& Lehmann, J. Some Xylaria species on termite nests. Mycologia 97, 914-923 (2005).

70 Sands, W. A. The initiation of fungus comb construction in laboratory colonies of Ancistrotermes guineensis (Silvestri). Insectes Soc. 7, 251-263 (1960).

71 Thomas, R. J. Distribution of Termitomyces and other fungi in the nests and major workers of several Nigerian macrotermitinae. Soil Biol. Biochem. 19, 335-341 (1987).

72 Visser, A. A., Nobre, T., Currie, C. R., Aanen, D. K. \& Poulsen, M. Exploring the potential for Actinobacteria as defensive symbionts in fungus-growing termites. Microb. Ecol. 63, 975-985 (2012).

73 Carr, G. et al. Microtermolides A and B from termite-associated Streptomyces sp. and structural revision of vinylamycin. Org. Lett. 14, 2822-2825 (2012).

74 Bai, R., Friedman, S. J., Pettit, G. R. \& Hamel, E. Dolastatin 15, a potent antimitotic depsipeptide derived from Dolabella auricularia: interaction with tubulin and effects on cellular microtubules. Biochem. Pharmacol. 43, 2637-2645 (1992).

75 Linington, R. G. et al. Antimalarial peptides from marine cyanobacteria: isolation and structural elucidation of Gallinamide A. J. Nat. Prod. 72, 14-17 (2009).

76 Molinski, T. F., Reynolds, K. A. \& Morinaka, B. I. Symplocin A, a linear peptide from the bahamian cyanobacterium Symploca sp. configurational analysis of N,N-Dimethylamino acids by chiral-phase HPLC of naphthacyl esters. J. Nat. Prod. 75, 425-431 (2012)

77 Luesch, H. et al. Symplostatin 3, a new Dolastatin 10 analogue from the marine cyanobacterium Symploca sp. VP452. J. Nat. Prod. 65, 16-20 (2002). 